\title{
Multispectral labeling technique to map many neighboring axonal projections in the same tissue
}

\section{Citation}

Tsuriel, Shlomo, Sagi Gudes, Ryan W Draft, Alexander M Binshtok, and Jeff W Lichtman. 2015. "Multispectral Labeling Technique to Map Many Neighboring Axonal Projections in the Same Tissue." Nat Meth 12 (6) (April 27): 547-552. doi:10.1038/nmeth.3367.

\section{Published Version}

doi:10.1038/nmeth.3367

\section{Permanent link}

http://nrs.harvard.edu/urn-3:HUL.InstRepos:23526183

\section{Terms of Use}

This article was downloaded from Harvard University's DASH repository, and is made available under the terms and conditions applicable to Other Posted Material, as set forth at http:// nrs.harvard.edu/urn-3:HUL.InstRepos:dash.current.terms-of-use\#LAA

\section{Share Your Story}

The Harvard community has made this article openly available.

Please share how this access benefits you. Submit a story.

Accessibility 


\title{
Multispectral labeling technique to map many neighboring axonal projections in the same tissue
}

Shlomo Tsuriel ${ }^{1,2,3,4}$, Sagi Gudes ${ }^{3,4}$, Ryan W. Draft ${ }^{1,2}$, Alexander M. Binshtok ${ }^{3,4}$, Jeff W. Lichtman $^{1,2}$

${ }^{1}$ Center for Brain Science, Harvard University; ${ }^{2}$ Department of Molecular and Cellular Biology, Harvard University, Cambridge MA USA 02138; ${ }^{3}$ Department of Medical Neurobiology, Institute for Medical Research Israel Canada Faculty of Medicine, ${ }^{4}$ The Edmond and Lily Safra Center for Brain Sciences, The Hebrew University, Jerusalem, 91120, Israel.

Correspondence should be addressed to A.M.B (alexander.binshtok@ekmd.huji.ac.il) and J.W.L. (ieff@mcb.harvard.edu)

\begin{abstract}
We describe a method to map the location of axonal arbors of many individual neurons simultaneously based on the spectral properties of retrogradely transported dye-labeled vesicles. We inject overlapping regions of an axon target area with three or more different colored retrograde tracers. Based on the combinations and intensities of the colors in the individual vesicles transported to neuronal somata we calculate the projection sites of each neuron's axon. This neuronal positioning system (NPS) enables mapping of many axons in a simple automated way. NPS combined with spectral (Brainbow) labeling of the input to autonomic ganglion cells show that the locations of ganglion cell projections to a mouse salivary gland relate to the identities of their preganglionic axonal innervation. We also show that NPS can delineate projections of many axons simultaneously in the mouse CNS.
\end{abstract}




\section{Introduction:}

Describing neuronal connectivity in detail is crucial for understanding of how neuronal circuits work, but challenging because most neurons not only receive input from multiple sources distributed over a large portion of the nervous system but also send signals to target cells that are widely distributed. Progress has been made using retrograde transsynaptic labeling approaches to identify the cohorts of neurons that are presynaptic to a given neuron ${ }^{1,2}$. Localizing all the postsynaptic targets of a neuron has been more difficult. Most retrograde labeling techniques show all the neurons that project to a particular place but not all the places a neuron projects to. Occasionally by use of multiple dyes injected into different regions, the presence of doubly or triply colored retrogradely labeled cells shows that a neuron projects to two or three different regions $^{3-6}$. These methods however do not have enough resolution to show for example how much of an axon arbor is distributed to each of its target regions or how multiple neurons that project to the same regions differ in their projections and therefore the answer to the "big question" of how the arbors of many different axons of the same class are organized relative to each other is largely unknown.

We have been interested in studying the organization of axonal projections among individual members of a single class of neurons. In a very thin rodent muscle this can be done in a painstaking way by reconstructing the arbors of many overlapping neurons by creating a high resolution confocal montage of the entire set of arbors of all the axons and then reconstructing each $^{7}$. But this approach does not scale well; as the number of axons being studied increases and the areal spread enlarges, the time to complete such a reconstruction becomes too long and the sizes of the datasets become unmanageable.

Here we use a multi-color retrograde labeling method that we developed to define individual axonal arbors that does not require tracing out each branch of each axon. The technique scales well because each of many nearby neurons in the same tissue sample can be analyzed by imaging their somata and imaging the target field but nothing in between. To do this mapping we inject 3-4 differently colored probes into partially overlapping contiguous regions of a projection area. Rather than look at the color of the neuron's soma in composite, we determine the spectral 
properties of each of many retrogradely transported vesicles in a cell body. We show that the color of each vesicle in the cell body maps to a site in the axon's projection. This mapping technique (which we call NPS, for neuronal positioning system) provides an easy method to get a large amount of detailed axon projection data without a great deal of work.

In this study we first examine axonal projections in the mouse parasympathetic submandibular ganglion, which drives saliva secretion from the submandibular gland. This neural system is interesting because of its simplicity and because dramatic changes in axonal connectivity occur during development ${ }^{8,9}$. The ganglion cell axons project into the submandibular gland but little is known about their organization. Using the NPS approach we define the axonal arborization area of individual ganglion cells and demonstrate that the projection territory of ganglion cell axons is linked to the particular presynaptic input each ganglion cell receives. Finally, we show that the NPS approach can be used in the central nervous system to map axonal projections between thalamus and cortex. 


\section{Results:}

\section{A method for mapping axonal projections of many neurons}

The goal of these experiments is to use retrograde labeling to map positions of axon branches of individual neurons. We began by injecting spectrally different Alexa Fluor (AF) fluorescent dyes conjugated to Wheat Germ Agglutinin (WGA) to nearby locations in the submandibular salivary gland (Fig. 1a). Each of the dyes is injected in a single site within the gland and each then diffuses into surrounding regions. Because of the spread, different dyes overlapped at boundary areas between the injection sites (Fig. 1a). Between 16 and 20 hours later we acquire high resolution fluorescence confocal image stacks of submandibular ganglion cells that project to the gland. These images reveal multiple labeled neuronal somata and within each we observe fluorescently labeled vesicles (Fig. 1b). Given the colors, these vesicles must have been transported retrogradely from the injected areas of the gland. These vesicles vary both in terms of their intensities and color combinations even within single neurons (Fig. 1b, c).

We hypothesized that the reason for the color and intensity variability of the vesicles in a single cell is that vesicular uptake is local and reflects the dye combinations at the site of uptake. Given that an axon branches to several locations the dye combinations should be different at each site. We further surmise that the vesicles remain separate from each other during transport to the soma and then at least for a time remain distinct in the soma. To test these ideas, we analyzed the transport of two dyes (red and green tagged WGA) that were injected into non-overlapping regions of the gland. We found 6 ganglion cells that possessed both red and green vesicles in their somata (see for example cell i, Fig. 1b). 95.4\% of the vesicles (372/390) had red or green fluorescence that was unmixed (either purely red or purely green) which is consistent with the idea that the dyes did not mix by fusion of vesicles during either transport or processing in the soma (red and green areas in Fig. 1d, Supplementary movie 1). The small percentage (4.6\%) of vesicles that appeared to be both red and green (yellow area in Fig. 1d) were actually multiple vesicles that overlapped in the optical image as measured by the different center of gravity of the two colors (Fig. 1d). In contrast, when we injected a mixture of the colors to one site, the center of gravities for the two colors in each vesicle were nearly identical. Thus when axons extend into 
areas that have different colored dyes, the individual vesicles show no mixing. These results imply that when a vesicle is filled with more than one color of dye it is because the dyes are present in the same region of the gland. This idea is confirmed by the blue dye injection that was also carried out in this same experiment. It overlapped with both the green and the red regions and many vesicles contained both blue and green or both blue and red (cells i-iii, Fig. 1b-c).

In addition to different color mixtures in the gland (that are reflected in different color mixtures in vesicles), there were also regions of the gland where the dye intensities were different due to dilution based on their distance from sites of dye injection. If the amount of dye uptake in a vesicle by an axon is related to the concentration of the dye at the site of uptake, we would expect that some vesicles will be brighter than others. To test this idea we injected three regions of a gland with three different concentrations of WGA-AF488 (Fig. 2a) and co-injected a second different color at high concentration at each of these sites (Fig. 2b). We wished to know if the intensity of the dye in a region of the gland was directly proportional to the intensity of the same dye in the vesicles that took up the dye from that region. We could keep track of which injection was associated with which neurons' vesicles by noting the second color (different at each of the three injection sites, Fig. 2c-e). The results showed a linear relation between the dye intensity at injection sites and the dye intensity of vesicles that took up the dye from each of these sites (Fig. 2f). Moreover we saw no evidence of the presence of one color affecting the value of another color in the same vesicle. Therefore we conclude that vesicle color intensity combinations can be used to help determine the territory of a single axon branch. Moreover documenting the color combinations in all the vesicles in a neuronal soma should reflect the spatial distribution of the entire projection of an axon within a dye-labeled area of the target tissue.

The mapping strategy is based on knowing the unique color combination and relative color intensities of dyes at each region of the injected gland. For example, if a red, green, and blue dye are injected as shown in Figure 3a, different points will have distinct color combinations and intensities. Thus, axonal uptake from these different regions will generate differently colored vesicles that mimic the color variations in the target tissue (Fig. 3a). 
We use this retrograde labeling approach to map the axonal projections of many individual submandibular ganglion cell neurons that send their axons to the same gland target. We pressure injected 0.5-1.5 $\mu 1$ of wheat germ agglutinin (WGA) tagged with four different spectrally distinct fluorophores (AF488/555/594/647). The injections were made in 4 contiguous areas within the gland such that the concentrated WGA $\left(10 \mathrm{mg} \mathrm{ml}^{-1}\right)$ at each injection site diffused into nearby areas and overlapped with the sites of dye spillover from other dye injections (Fig. 3b). In the experiments described below, the 4 dye injections cover area of $\sim 1 \mathrm{~cm}^{2}$ or about $\sim 10 \%$ of the flattened gland surface.

We compared the color properties of 20-250 vesicles (mean of 78.8 \pm 3.1 ) in each labeled neuron (Fig. 3c) with the dye distributions in the gland, in order to locate the axonal projection of a neuron in the gland. This calculation is done in several steps. The first step is to assay the color intensities present in each vesicle in a neuron using a four-color confocal stack of images (in the case where 4 dyes are injected in the target). Second, for each color we normalize its intensities to the one vesicle in the entire set of ganglion cell clusters in an animal that exhibited the maximum intensity of that color (see Methods). Based on the linear relationship between the dye intensity at injection sites and the dye intensity of vesicles (Fig. 2f), we assume that the vesicle in the whole set of ganglion cell clusters with the maximum intensity for one color (which we give an intensity of 1) must come from an axon branch at the site where the dye intensity in the gland is maximal. Third, we normalize the gland dye intensities to the sites of maximum intensity for each dye just as was done for intensities of vesicles in ganglia.

We use the four color values within each vesicle to find the site where the dye-injected gland best matches the vesicle's color intensities (white spot, Fig. 3d). In this way we map every vesicle to the site in the gland that most strongly correlates with its intensity profile. We then repeat the same procedure for all of the vesicles in a neuron to map the territory of its axonal arbor within the gland (Fig. 3e). The composite result for all the vesicles in a ganglion cell generates a projection that is usually 15-25 times larger than the area associated with a single vesicle. The territory occupied by a neuron is not usually homogeneous but clusters into a number of small areas, a pattern that is both in size and organization, similar to the axonal arbors 
of transgenically labeled submandibular ganglion cells that terminate in multiple small baskets

(Sheu, S, Tapia JC and JWL, unpublished data). This similarity suggests that axons are collecting dye mostly from their terminal branches.

This whole procedure was accomplished by an automatic algorithm (see Supplementary MATLAB script) that allowed analysis of multiple labeled ganglion cells in a cluster (Fig. 3f). As can be seen from this analysis, some neurons had non-overlapping territories (e.g. cells iv and v, Fig. 3f) but different neurons sometimes mapped to nearly identical regions (e.g., cells iii and v, Fig. 3f).

In summary, this method calculates axonal arbor positions based on the relation between vesicle color and the contours of the intensity profiles surrounding several differently colored injections in a target tissue. This strategy is in some ways analogous to the principle used in a global positioning system (GPS) receiver which uses distances from 3 or more satellites to triangulate its position. For this reason we call this technique a Neuronal Positioning System (NPS).

\section{Mapping two levels in the same synaptic circuit}

To study the projections at two levels of a synaptic circuit in the peripheral nervous system we have applied the NPS method in Brainbow transgenic mice ${ }^{12,13}$. We have used Thy-1 membrane-tagged Brainbow construct (Mem-Brainbow) that labels nearly all of the preganglionic axons in a variety of different colors (Fig. 4a and b). Mem-Brainbow however is not expressed in ganglion cells, so the vesicle colors are not contaminated with brainbow expression in ganglion cells. This approach allowed us to determine if there was any relation between the axonal projections of postganglionic neurons and their upstream preganglionic innervation. In adult submandibular ganglia, preganglionic axons establish basket-like terminals on the somata of ganglion cells $\mathrm{s}^{8,14}$. We studied the axonal projections based on retrogradely labeled vesicles of ganglion cells in clusters of 20-80 neurons in young adult (P32) Mem-Brainbow animals (Fig. 4a). Due to Brainbow expression, we also studied the preganglionic input to these same cells (Fig. $4 \mathbf{b}$ and c). We measured the distance between the centers of gravity of the axonal projections of pairs of ganglion cells that were innervated by the 
same preganglionic axon with pairs of ganglion cells innervated by different preganglionic axons. We found that each set of ganglion cells that was innervated by a single preganglionic axon tended to have their projections clustered in a circumscribed part of the gland and vice versa (Fig. 4dI-VI, e and f; Supplementary Fig. 1).

\section{Using NPS to map projections of many neurons in the CNS}

To see if this approach could also be used in the CNS, we studied the axonal projections of thalamocortical neurons into layer 4 of barrel cortex ${ }^{17,18}$. We pressure-injected WGA tagged with 4 different AF colors into adjacent overlapping areas of layer 4 barrel cortex in mice (Fig. 5a, b; the barrel boundaries were defined by the DAPI staining, data not shown). These injections created a roughly $1 \mathrm{~mm}^{2}$ region of dye in the posteromedial barrel subfield (Fig. 5b). Six to 12 hours later the dyes were visible in thalamic cell somata in the ventral posteromedial (VPM) nucleus (Fig. 5c). The thalamocortical neurons were labeled in a topographic manner that reflected the arrangement of dyes in the injected region of cortex (compare Fig. 5b and 5c). However the color boundaries were often much sharper in the thalamus than in the cortex. For example adjacent cells in one region were sharply partitioned into green and purple (see arrows Fig. 5c) with only a few cells sharing these colors suggesting that the intervening sites in layer 4 that contained the color mixture (green plus purple) were poorly innervated by thalamic axons from the examined area. This might be explained if axons from this thalamic area projected more often to barrels than to the septa between barrels (i.e. a barrelo-topic projection). Indeed, when we analyzed the projections of a set of thalamic neurons that fell at color boundaries we found that most of them projected to barrels rather than septa. We analyzed 23-130 vesicles (50.1 \pm 9.1 , $\mathrm{n}=13$ ) per neuron (see for example Fig. 5c, right panels) and used NPS to map their neuronal projections to layer 4 (Fig. 5d - g). The majority of the projections were within the borders of a single barrel (Fig. 5d, e). Some neurons (e.g. cell vi) had more than one projection area that covered more than one barrel and some neurons (e.g. cell viii) project more broadly to several barrels and in between them (Fig. 5d, e, see also Ref ${ }^{18}$ ). NPS also delineated, in a given barrel, the projections of different neurons (Fig. 5f) in the horizontal and vertical planes showing that 
these neurons projections largely overlap in the horizontal plane but differ in which sublayers of layer 4 that they project to (Fig. 5g, h). 


\section{Discussion:}

In this work we present an approach for mapping the axonal projections of each of many neurons in the same tissue at the same time. This technique should allow mapping of all the neurons in a large area in a relatively easy way. The method relies on analysis of the colors of individual dye-labeled vesicles in the somata of neurons whose axons project to a target area that has been blanketed with gradients of several different colors of dyes. We used fluorescent conjugates of WGA because of its efficiency as a retrograde label ${ }^{5,19,20}$. It is likely that other retrogradely transported agents could also be used but the WGA conjugates are relatively inexpensive and we have many color choices.

Because many neuronal somata could be studied in one tissue sample, this approach provided a means of mapping out the projections of many axons at the same time. To our knowledge there are no other techniques capable of this kind of detailed analysis of all the terminal branches of individual neurons in the same tissue sample.

We believe that quite large areas can be assessed with this approach. As shown in the salivary gland the dye filled target area is in the range of $1 \mathrm{~cm}^{2}$. In the CNS we injected an area that was about $1 \mathrm{~mm}^{2}$ but this could have been much larger. The size of the dye filled area can be increased by extending the distance between injection sites and increasing the injection volumes or by adding additional color channels (such as adding near UV dye and blue dye channels which were not used in the work we present here).

In conclusion, the ability to trace out the projections of many axons in the same piece of neural tissue offers an opportunity to learn about organizational principles that would otherwise be difficult or impossible to study. By seeing many axons in the same preparation one can ascertain the way neurons in one region (and of one type) partition or mix relative to each other. Using this method in the parasympathetic nervous system we found that axons that project to similar regions are innervated by the same presynaptic axon. This kind of inter-neuronal organization is probably commonplace in the nervous system but is invisible without methods to see each neuron as an individual entity rather than a member of a group of similarly labeled cells. Because 
this same labeling approach works well in the CNS, it offers the possibility of learning details of the organization of axonal projections in many situations. 


\section{Acknowledgments}

Support is gratefully acknowledged from the Humans Frontiers Science Foundation (S.T.), European Research Council (ERC) under the European Union's Seventh Framework Programme (FP7/2007-2013) / ERC grant agreement $n^{\circ} 260914$ (S.T., S.G. and A.B.), National Institute of

Mental Health Silvio Conte Center 1P50MH094271 (J.W.L.) and National institute of Health NS076467 (J.W.L.). We thank R. Kafri for helping generating the MATLAB code.

\section{Author contribution statements:}

S.T. conceived the NPS strategy, designed and conducted the experiments, analyzed the data, and wrote the manuscript. S.G. conducted the experiments, analyzed the data and wrote the manuscript, R.W.D. created Mem-Brainbow mice and wrote the manuscript. A.M.B. designed the experiments, analyzed the data, supervised the project and wrote the manuscript. J.W.L. conceived the study, designed the experiments, analyzed the data, supervised the project and wrote the manuscript.

Statement of Competing Financial Interests: The authors declare no competing financial interests. 


\section{Reference List}

1. Wickersham, I. R., Finke, S., Conzelmann, K.-K. \& Callaway, E. M. Retrograde neuronal tracing with a deletion-mutant rabies virus. Nat. Methods 4, 47-9 (2007).

2. Wickersham, I. R. et al. Monosynaptic restriction of transsynaptic tracing from single, genetically targeted neurons. Neuron 53, 639-47 (2007).

3. Hörner, M. \& Kümmel, H. Topographical representation of shoulder motor nuclei in the cat spinal cord as revealed by retrograde fluorochrome tracers. J. Comp. Neurol. 335, 309-19 (1993).

4. Bentivoglio, M. \& Molinari, M. Fluorescent retrograde triple labeling of brainstem reticular neurons. Neurosci. Lett. 46, 121-126 (1984).

5. Reeber, S. L., Gebre, S. a \& Sillitoe, R. V. Fluorescence mapping of afferent topography in three dimensions. Brain Struct. Funct. 216, 159-69 (2011).

6. Sincich, L. C., Jocson, C. M. \& Horton, J. C. V1 interpatch projections to v2 thick stripes and pale stripes. $J$. Neurosci. 30, 6963-6974 (2010).

7. Lu, J., Tapia, J. C., White, O. L. \& Lichtman, J. W. The interscutularis muscle connectome. PLoS Biol. 7, e32 (2009).

8. Lichtman, B. Y. J. W. and Biophysics, Washington University School. 155-177 (1977).

9. Snider, D. The Dendritic Complexity and Innervation in Five Species of klamkals of Submandibular Neurons. 7, (1987).

10. Bolte, S. \& Cordelières, F. P. A guided tour into subcellular colocalization analysis in light microscopy. J. Microsc. 224, 213-32 (2006).

11. Tapia, J. C. \& Lichtman, J. W. in Fundam. Neurosci. (Squire, L. R.) 437-530 (Academic Press, 2013).

12. Livet, J. et al. Transgenic strategies for combinatorial expression of fluorescent proteins in the nervous system. Nature 450, 56-62 (2007).

13. Cai, D., Cohen, K. B., Luo, T., Lichtman, J. W. \& Sanes, J. R. Improved tools for the Brainbow toolbox. Nat. Methods 10, 540-7 (2013).

14. McCann, C. M., Tapia, J. C., Kim, H., Coggan, J. S. \& Lichtman, J. W. Rapid and modifiable neurotransmitter receptor dynamics at a neuronal synapse in vivo. Nat. Neurosci. 11, 807-15 (2008).

15. Lichtman, B. Y. J. W. Biophysics, Washington University. 121-130 (1980).

16. Coggan, J. S. et al. Age-associated synapse elimination in mouse parasympathetic ganglia. J. Neurobiol. 60, 214-26 (2004).

17. Agmon, A. \& Connors, B. W. Thalamocortical responses of mouse somatosensory (barrel) cortex in vitro. Neuroscience 41, 365-379 (1991).

18. Oberlaender, M. et al. Cell type-specific three-dimensional structure of thalamocortical circuits in a column of rat vibrissal cortex. Cereb. Cortex 22, 2375-2391 (2012). 
19. Gonatas, N. K., Harper, C., Mizutani, T. \& Gonatas, J. O. Superior sensitivity of conjugates of horseradish peroxidase with wheat germ agglutinin for studies of retrograde axonal transport. J. Histochem. Cytochem. 27, 728-734 (1979).

20. Wan, X. C., Trojanowski, J. Q. \& Gonatas, J. O. Cholera toxin and wheat germ agglutinin conjugates as neuroanatomical probes: their uptake and clearance, transganglionic and retrograde transport and sensitivity. Brain Res. 243, 215-24 (1982).

21. Oh, S. W. et al. A mesoscale connectome of the mouse brain. Nature 508, 207-14 (2014).

22. Hunnicutt, B. J. et al. A comprehensive thalamocortical projection map at the mesoscopic level. Nat. Neurosci. 17, 1276-1285 (2014).

23. Zingg, B. et al. Neural networks of the mouse neocortex. Cell 156, 1096-1111 (2014).

24. Langley, J. N. On the Origin from the Spinal Cord of the Cervical and Upper Thoracic Sympathetic Fibres, with Some Observations on White and Grey Rami Communicantes. Philos. Trans. R. Soc. B Biol. Sci. 183, xviii-124 (1892).

25. Langley, J. N. in Textb. Physiol. (SCHAFER, E. A.) 616-686. (Pentland, 1900).

26. Lichtman, B. Y. J. W., Purves, D., Yip, J. W., Avenue, S. E. \& Louis, S. Washington University. 69-84 (1979).

27. Shaner, N. C. et al. Improved monomeric red, orange and yellow fluorescent proteins derived from Discosoma sp. red fluorescent protein. Nat. Biotechnol. 22, 1567-72 (2004).

28. Zacharias, D. a, Violin, J. D., Newton, A. C. \& Tsien, R. Y. Partitioning of lipid-modified monomeric GFPs into membrane microdomains of live cells. Science 296, 913-6 (2002).

29. Rizzo, M. a, Springer, G. H., Granada, B. \& Piston, D. W. An improved cyan fluorescent protein variant useful for FRET. Nat. Biotechnol. 22, 445-9 (2004).

30. Kay, J. N. et al. Transient requirement for ganglion cells during assembly of retinal synaptic layers. Development 131, 1331-42 (2004).

31. Caroni, P. Overexpression of growth-associated proteins in the neurons of adult transgenic mice. J. Neurosci. Methods 71, 3-9 (1997).

32. Dewachter, I. et al. Neuronal deficiency of presenilin 1 inhibits amyloid plaque formation and corrects hippocampal long-term potentiation but not a cognitive defect of amyloid precursor protein [V717I] transgenic mice. J. Neurosci. 22, 3445-53 (2002).

33. Paxinos, G. \& Franklin, K. B. J. The mouse brain in stereotaxic coordinates. Acad. Press 2nd, 138 (2004).

34. Binshtok, A. M., Fleidervish, I. A., Sprengel, R. \& Gutnick, M. J. NMDA receptors in layer 4 spiny stellate cells of the mouse barrel cortex contain the NR2C subunit. $J$. Neurosci. 26, 708-715 (2006).

35. Fleidervish, I. A., Binshtok, A. M. \& Gutnick, M. J. Functionally distinct NMDA receptors mediate horizontal connectivity within Layer 4 of mouse barrel cortex. Neuron 21, 1055-1065 (1998). 


\section{Figure Legends}

Figure 1. Colors of retrogradely transported vesicles reflect a neuron's axonal projection. (a) The submandibular gland was injected with three differently colored WGA conjugated fluorophores (inset, see Methods for details). Image shows the strategy of injecting differently colored dyes in contiguous overlapping territories. Note that the red label and the green label territories do not overlap with each other but both overlap with the blue label (scale bar $=$ $500 \mu \mathrm{m})$. (b) About 16 hours after the gland was labeled clusters of submandibular ganglion cells imaged with confocal microscopy exhibited multiple colored vesicles. Each dotted line outlines of a separate ganglion cell soma. From these collapsed stacks ( 20 images per soma) it can be seen that cells have a wide variety of differently colored vesicles. Many of these colors are mixtures of the primary colors injected into the gland. Note that there are no vesicles in this image that have mixtures of green and red (i.e. shades of yellow) but many that are cyan (green plus blue) or purple (red plus blue) consistent with the overlap of labels in the gland. Interspersed with the labeled ganglion cells are many cells with much less or no labeling as would be expected from dye injections that cover a small portion of a gland's volume (scale bar $=10 \mu \mathrm{m})$. (c) Single confocal planes of the three numbered cells in panel $b$. (d) Vesicle intensities from 6 cells following two label injections into non-overlapping regions of the gland. A small number 2-3 vesicles per cell, (4.6\%) itemized by the automatic algorithm (in the yellow region of the graph) had both of the colors (that were injected in non-overlapping regions of the gland) above threshold. However as shown in the inset, these "vesicles" were actually superimpositions of vesicles that happened to be located near each other (see Supplementary movie 1). The automatic algorithm rejects vesicles that are larger than diffraction limited spots (see Methods) and this removes many ambiguous vesicles, such as the leftmost column in which two vesicles are contiguous but have different centers of gravity (see Methods).

Figure 2. The intensity of retrogradely labeled vesicles is a linear measure of the label intensity at axonal uptake sites. (a, b) A submandibular gland labeled with a high medium and low concentration of WGA-Alexa 488 (a) These injections were pre-mixed with WGA conjugated to 3 different fluorophores respectively (b) Scale bar $=500 \mu \mathrm{m}$. (c-e) Left panels shows the different 
intensities of Alexa488 in ganglion cells from the three different injection sites confirmed by the highest intensity labeling of any ganglion cells for each of the three co-mixed colors (right panels). Scale bar $=10 \mu \mathrm{m}$. (f) A comparison of the vesicle and gland dye intensity for 12 ganglion cells from 4 ganglia shows a linear relation between the intensity in the gland to the intensity of vesicles that are transported back to the cell soma. Dashed line shows the optimal linear relation $(\mathrm{r}=.98)$.

Figure 3. A Neural Positioning System (NPS) for mapping axonal projections. (a) Diagram illustrating the rationale of the mapping technique. Multiple partially overlapping different colored labels in a target tissue (bottom) generate small areas in the target that have unique color properties. For example, a point in the gland near the blue injection site will have a high concentration of blue dye, a small concentration of red dye, and an intermediate concentration of green dye . A different point might have more similar concentrations of the blue and red dye but a greater concentration of the green dye. A neuron that takes up vesicles whose intensity and color mixtures match the sites of uptake, will have in it soma a range of vesicle colors and intensities (top middle). In the diagrammatic example shown, two vesicles are highlighted (arrows) whose color combinations match small regions in the gland. By finding these matches for each vesicle, the projection of a neuron can be mapped automatically. (b) Submandibular gland (P32) showing the injection sites of WGA conjugated to the 4 color fluorophores (AF 488 - green, AF 555 - blue, AF 594 - red, AF 647 - yellow). Red rectangle marks the labeled area analyzed in D - F. Scale bar $=1 \mathrm{~mm}$. (c) Ganglion cells that project to the dye labeled gland region. Scale bar $=10 \mu \mathrm{m}$. (d) The mapping of one vesicle (from neuron ii in panel $c$ ) to the gland region is based on locating the pixels in the gland whose 4 color properties best match (top $0.0001 \%$, see Methods) the color properties of the vesicle. Inset shows a magnified version of the gland volume denoted by the white box. The single pixel in the gland with the best match is shown in black in the inset (see arrow, Scale bar inset $=25 \mu \mathrm{m}$ ). The regions bordered by a black outline are the pixels above the threshold. (e) Mapping all the vesicles of a neuron (cell ii in panel $c$ ) requires repeating the process described in panel $d$ for each vesicle. Each white dot represents the vesicle matches above the cutoff threshold. Shown is the outline of the contiguous 
region in the gland that best matched the range of colors of all the vesicles of neuron 2 in panel $c$.

(f) Analysis of the projections 5 cells from panel $c$. Note that cell iii and $\mathbf{v}$ have similar projections, whereas cells i, ii and iv do not overlap with them at all. Scale bar $d-f=500 \mu \mathrm{m}$.

Figure 4. Ganglion cells innervated by the same preganglionic axon project to nearby regions in the gland. (a) A P32 submandibular ganglion cell cluster back-labeled with fluorescent dyes injected into the submandibular gland. (b) To see the preganglionic input to these cells we did the experiment in a brainbow transgenic mouse line in which individual preganglionic axons are labeled with different combinations of three fluorescent proteins. Confocal reconstructions thought the cluster allow us to distinguish which ganglion cells are innervated by each separately colored preganglionic axon. The brainbow labels are less intense than the Alexa fluor-labeled retrograde vesicles so that the gain can be turned down to the point where only the vesicles are visible (see panel $a$ ). The brainbow colors also use a color that was not used in the retrograde labeled signal. For these reasons and the different localization of preganglionic axons that surround ganglion cells versus ganglion cells per se, is was not difficult to disambiguate the preganglionic colors from the ganglion cell soma colors. (c) Rendering showing each of the preganglionic axons and the identity of each ganglion cell based on data in panel $a$ and $b$. The circle in each ganglion cell body represents the color of the preganglionic axon or axons that innervate it. Scale bar $a-c=10 \mu \mathrm{m}$. (d, I-VI) Projections of cohorts of ganglion cells based on the composite projections of all the neurons innervated by each of 5 different preganglionic axons. For example, $d I$ shows the projections of neurons 2, 4, 7, and 9 (panel $c$ ) that are all innervated by the "blue" preganglionic axon. As can be seen each preganglionic axon drives neurons in overlapping but not identical regions of the gland. The superimposition of all these projections are shown in VI. (e) Higher magnification version of panel $d V I$. Rather than random projections preganglionic axons drive an orderly arrangement of territories in the gland, despite the fact that ganglion cells innervated by a preganglionic axon are not necessarily contiguous in the ganglion cell cluster (see panel $c$ ). (f) Diagram showing the centers of gravity of the ganglion cell projections of each of the 19 cells shown in panels $a-c$. The color of the circles represent the 
identity of the innervating preganglionic axon. The locations are superimposed on the labeled gland image. Note the high frequency that centers of gravity are similar when the ganglion cells are innervated by the same preganglionic axon (i.e., colored circles are often near other circles of the same color). Scale bar $d-f=500 \mu \mathrm{m}$.

Figure 5. NPS mapping of the thalamic projection to barrel cortex (a) $\sim 20 \mathrm{nl}$ of WGA conjugated to 4 different AF colors was injected into nearby overlapping regions of layer 4 of the barrel cortex of adult mice ( $\mathrm{n}=7$, one example shown here). Each panel shows the maximum projection of one of the injected fluors (bar $=200 \mu \mathrm{m}$ ). (b) Merged image of the 4 color injections superimposed on the location of the barrels shown as dotted lines, based on DAPI stain - see methods (bar $=200 \mu \mathrm{m})$. (c) The retrogradely labeled area in the VPM of the thalamus. Shown is one $100 \mu \mathrm{m}$ thick section of 7. Note that the organization of the colors in the thalamus corresponds to the locations of injection sites in the cortex $(\mathrm{bar}=100 \mu \mathrm{m})$. Eight thalamic somata are shown on the right side of the panel at higher magnification. Each neuron's axonal projection was mapped using NPS. (bar in right side of panel $=5 \mu \mathrm{m}$ ). (d) On the background of the labeled cortical area (see panel b) we show the axonal centers of gravity for each of the labeled neurons in the right side of panel $\mathrm{c}$. Note that neuron vi has two centers of gravity because it projects to two distinct sites ( $\mathrm{bar}=200 \mu \mathrm{m}$ ). (e) Cortical projection outlines for each neuron were rendered as in previous figures. Most of these projections aligned well with barrels although several either projected to regions between barrels or projected to more than one barrel (bar $=200 \mu \mathrm{m}$ ). (f) High magnification of the area outlined by the red rectangle in panel e. Note that even though the 3 arbors overlap in the same barrel, each one has a different shape (bar $=$ $100 \mu \mathrm{m})$. (g) Rendering of the three axonal projections shown in $\mathrm{f}$ in the $\mathrm{X}-\mathrm{Z}$ plane (maximum projection of the $Y$ axis). Dashed line illustrates the barrel boundary (vertical bar $=50 \mu \mathrm{m}$; horizontal bar $=100 \mu \mathrm{m})$. (h) Each of the three projections shown separately indicates that they project to somewhat different sublayers of layer 4 (calibration as in panel g). 


\section{Online Methods:}

\section{Animals and labeling}

All animal experiments were approved by the Faculty of Arts and Sciences Institutional Animal Care and Use Committee of Harvard University or the Ethics committee of Hebrew University. Male and female C57 black mice were anesthetized with $100 \mathrm{mg} \mathrm{kg}^{-1} \mathrm{ketamine}$ and $10 \mathrm{mg} \mathrm{kg}^{-1}$ xylazine. The ventral neck hair was then removed (using a commercial hair removal cream) and a 1-3 cm midline incision was made from the base of the chin to the sternum to expose the submandibular glands bilaterally. To inject the gland(s) with dyes, we used a pulled glass pipette attached to a syringe. The injections were done in the lateral lower third of one or both glands using hand pressure to a syringe after inserting the pipette several tens of microns beneath the gland capsule. At each injection site $\sim 0.5 \mu 1$ of $10 \mathrm{mg} \mathrm{ml}^{-1}$ wheat germ agglutinin (WGA) conjugated to Alexa fluor (AF, Invitrogen) 488, 555, 594, or 647 was injected. In principle to localize a point on a 2 dimensional surface, 3 colors are sufficient and for mapping a 3 dimensional space, no more than 4 colors are needed. Although the salivary gland is three dimensional, we restricted the injections to regions near the gland's surface where imaging the dye concentrations is more straightforward due to less scatter. The areas that we studied were restricted to a $150 \mu \mathrm{m}$ rind near the gland surface and we kept track of the $\mathrm{X}, \mathrm{Y}$ and $\mathrm{Z}$ coordinates of the dye spread. We found as expected that when we ignored all but one color, the positioning result of each vesicle degraded. The precision of vesicle mapping to the gland improved progressively with each additional color channel up to the 4 we tried (data not shown). The injections within each gland were spaced $\sim 1 \mathrm{~mm}$ apart from each other in a square-shaped pattern. After the injections, the ventral skin was sutured and the animal monitored until surgical recovery and provided with an injection of Buprenorphine $\left(0.1 \mathrm{mg} \mathrm{kg}^{-1}\right)$ for pain management. Because vesicles that are transported back to the soma eventually enter larger organelles in the lysosomal pathway and get degraded (at $\sim 1$ week after dye injection there is no color in the ganglion cell somata), we did all imaging between 16-20 h post target injection when the vesicles had just appeared in the ganglion cell somata and well before the lysosomal process had removed them. The animals were re-anesthetized and perfused transcardially with cold $\left(4^{\circ} \mathrm{C}\right)$ fixative 
solution containing 4\% paraformaldehyde and $120 \mathrm{mM}$ sucrose in PBS. The gland, ganglion tissue and duct were then extirpated. To prepare for histological study, the gland was pressed between two glass slides in fixative solution for 3 hours. The duct was incubated for $1 \mathrm{hr}$ in fixative solution and for another hour in fixative solution with 1\% Triton X100.

A subset of the mice used in this study also expressed a new Brainbow transgene (Mem-Brainbow), resulting in fluorescent labeling of $\sim 90 \%$ of preganglionic axons that project to the submandibular ganglion. To make this transgenic line, we first cloned a CMV-Brainbow 1.0 style construct using membrane bound fluorescent proteins and pairs of incompatible Lox $\mathrm{P}$ and Lox 2272 sites (as described in Livet et al. 2007). The construct encodes (5' to 3') mCherry $^{27}$, monomeric EYFP ${ }^{28}$, and monomeric Cerulean ${ }^{29}$. A short palmitolaytion sequence ${ }^{30}$ was fused to a linker at the N-terminus of each protein. The Thy1 Mem-Brainbow construct was generated by blunt cloning a Nhe1/Scal fragment containing the Brainbow coding region (3.4 $\mathrm{kb})$ into the Xho1 site of the Thy1.2 cassette $^{31}$. Mem-Brainbow mice were crossed with a Thy1-Cre transgenic line ${ }^{32}$ to switch expression from default mCherry to Cerulean or EYFP.

Injection to the barrel cortex: WGA-conjugated to AF dyes 488, 555, 594, or 647 were stereotactically injected into Layer 4 of the primary somatosensory barrel cortex of 6-8 week old C57BL/6 mice, using pulled glass pipettes attached to a syringe. Injections were carried out under isoflurane anesthesia (2\%). Anesthetized mice were placed in a stereotaxic frame (David Kopf instruments, CA) and maintained on a heating blanket at $37^{\circ} \mathrm{C}$. The animal's heads were shaved, and a midline incision was made in the scalp to identify the Bregma and the Lambda. The location of the barrel cortex and the injection sites were estimated using the known stereotaxic coordinates $^{33}$ (for mice with 4.1 Bregma-Lambda distance as follows : - $0.8 \mathrm{~mm}$ AP from the Bregma; $3.1 \mathrm{~mm}$ lateral from the Bregma; $0.56 \mathrm{~mm}$ ventral to the skull surface). Bregma-Lambda distance was measured in each animal and adjustments were made accordingly. Using a small dental drill to allow pipette insertion, 4 holes were drilled $100 \mu \mathrm{m}$ posterior, anterior, medial and lateral to the above coordinates. Approximately $20 \mathrm{nl}$ of each dye was injected into each hole. The injection pipette was left at each injection site for 10 minutes. After the injections, the skin was sutured and the animal monitored until awake and provided with an 
injection of Buprenorphine $(0.1 \mathrm{mg} \mathrm{kg}-1)$ for pain management. Six to eight hours following the injections the mice were re-anesthetized and perfused transcardially with $20 \mathrm{ml}$ phosphate buffered saline followed by $15 \mathrm{ml}$ of 4\% paraformaldehyde (PFA). Brains were removed and postfixed in $4 \%$ PFA at $4{ }^{\circ} \mathrm{C}$ for 6 hours and then mounted in $1 \%$ agarose. A single slice containing Layer 4 of somatosensory cortex was obtained by pre-cutting the brains in the tangential plane as previously described ${ }^{34,35}$. In brief this was accomplished by trimming the base of the brain at a $30^{\circ}$ angle relative to the horizontal plane and at a $10^{\circ}$ angle relative to the vertical plane. The block was then oriented in a microtome (Leica V1000) with the trimmed surface down, so that the plane of the knife was tangential to the pial surface of the barrel field. The first $200 \mu \mathrm{m}$ slice, which contained Layers 1 and 2 and most of Layer 3, was discarded. The subsequent $400 \mu \mathrm{m}$ slice, in which the posteromedial barrel subfield was clearly visible using DAPI staining, was taken for the analysis. The remaining brain block was sliced coronally in $100 \mu \mathrm{m}$ sections and the ventral posteromedial (VPM) nucleus and posteromedial complex (PoM) of thalamus was identified using mouse brain maps.

\section{Imaging}

The target tissue was imaged on a laser scanning confocal (Zeiss LSM 7 or Leica SP5) using a 10X, 0.3 NA air objective at 2.8 X 2.8 X $5 \mu \mathrm{m} /$ voxels in the gland and $1.5 \mathrm{X} 1.5 \mathrm{X} 4 \mu \mathrm{m} /$ voxels in the barrel cortex. Retrogradely labeled ganglia of thalamus were imaged with a $63 \mathrm{X}, 1.4 \mathrm{NA}$ oil objective in $0.09 \times 0.09 \times 0.4 \mu \mathrm{m} /$ voxel. We used the $488 \mathrm{~nm}$ Argon laser to excite AF 488 (490 - $523 \mathrm{~nm}$ emission); a $561 \mathrm{~nm}$ photodiode laser (562-584 nm emission) was used for AF 555; a $594 \mathrm{~nm}$ photodiode laser (597-632 nm emission) for AF 594; and a $633 \mathrm{~nm}$ photodiode laser (659 - $740 \mathrm{~nm}$ emission) for AF 647. To image the brainbow colors in the preganglionic axons, for CFP we used $440 \mathrm{~nm}$ photodiode laser (459-487 $\mathrm{nm}$ emission) and for EYFP we used the $514 \mathrm{~nm}$ Argon laser (516 - $544 \mathrm{~nm}$ emission). To enhance the intensity of mCherry we used previously described rabbit anti-mCherry antibody ${ }^{13}$ (1:1000) that was bound to a secondary anti-rabbit antibody coupled to AF 633. For imaging AF 633 we used a $633 \mathrm{~nm}$ photodiode laser (640 - 694 emission). Each channel was imaged sequentially. WGA-AF fluorescence was much brighter than the intrinsic XFP brainbow fluorescence. Although the brainbow and the AF 
channels overlap they are distinguishable by their different location (preganglionic axons surrounding ganglion cell bodies versus retrogradely vesicles inside the ganglion cell body).

\section{Image analysis}

NPS analyses, as executed in this paper, are based on the assumption that for each dye the sites of maximal dye concentration in the target area is in fact innervated by at least some neurons in the presynaptic population.

In order to reduce noise we used a 2 pixel radius Gaussian filter on the target confocal images and 1 pixel radius filter on the ganglion or thalamus. Each analyzed soma was separated into a $3 \mathrm{D}$ cube that contained most of it retrogradely transported vesicles $(>90 \%)$ and more importantly, contained no vesicles from any other cell as these would contaminate the interpretation. To get the location of each vesicle we used the brightest pixel value among the 4 color channels of the ganglion somata images. These brightest values were merged into one 3D image that contain only the maximal values from all channels for each pixel in each image of the stack. We used the Imagej 3 dobject counter plugin ${ }^{10}$ to threshold the vesicles and localize them in 3D space. We excluded fluorescent spots that were noise because they were smaller than the diffraction limit (occupied less than 10 voxels $\left(0.039 \mu \mathrm{m}^{3}\right)$ or larger than the diffraction limit (50 voxels $=0.19 \mu \mathrm{m}^{3}$ (as more than one vesicle). In all experiments the detection of vesicles was done blindly to the vesicle original color.

Using the coordinates that localized the vesicles (see above) we then obtain the maximal value from each channel for each vesicle. Next, these values are normalized to the brightest vesicle value in the entire ganglion for each color channel. Using a custom MATLAB script (Supplementary MATALB script) we studied each vesicle sequentially by comparing its normalized value in each of the four color channels to the normalized values of the color channels at every position in the gland. The comparison was accomplished by generating a difference value for each vesicle color with each gland pixel. These 4 differences were then summed for each gland pixel:

$$
\left|V_{\text {green }}-G_{\text {green }} n\right|+\left|V_{\text {yellow }}-G_{\text {yellow }} n\right|+\left|V_{\text {orange }}-G_{\text {orange }} n\right|+\left|V_{\text {red }}-G_{\text {red }} n\right|
$$


Where $V$ is the normalized vesicle value for a color channel; green, yellow, orange and red are the different channels. $G$ is the normalized gland value for a color channel at pixel $n$. The pixel in the gland with the minimal sum of the differences is the pixel whose composite 4 color distribution best matches that vesicle's 4 color distribution (i.e., the best fit). In order to reduce noise for each vesicle we marked the $0.0001 \%$ of best fit pixels as the possible origin for the vesicle. In most cases these gland pixels were localized to the same area in the gland. To create the locale of the axonal projection of a single ganglion cell, we drew the perimeter surrounding the best fit pixels for all of the vesicles in the ganglion cell and circling 10-15 pixel radius around each that typically generated one or at most several contiguous regions.

To quantitate the size of these arbors, we use a measure of arbor length which is the distance between the two most geometrically distant points in the arbor. Arbor area and length were calculated on a 2 dimensional image that was created by summing best fitted points from the 3D image. We also calculated the arbor center of gravity using a MATLAB script that averaged the coordinates of all the fit pixels in the $\mathrm{X}, \mathrm{Y}$ and $\mathrm{Z}$ axes. The center of gravity was used to calculate the distances between pairs of neuron's arbors. To obtain a measure of the overlap of one arbor with any other, we counted the number of voxels that the arbor had in common with another arbor and divided that number by the total voxels in the arbor of interest. One potential confound in this analysis is that in older and larger mice, the gland size is proportionally larger. To compensate for different sized glands at different ages, we used only neuronal projections whose centers of gravity differed by no more than $10 \%$ of the gland length.

\section{Statistics}

For all statistical comparisons we used the unpaired one and two-sided student t-test corrected for the multiple comparisons, and one way ANOVA $*=\mathrm{p}<0.05 * *=\mathrm{p}<0.01 * * *=\mathrm{p}<0.001$, with post-hoc Bonferroni correction. Data represented as means \pm S.E.M. F-tests were used to examine the similarity of the variance of the compared groups and the appropriate t-tests were performed accordingly. 


\section{A multispectral labeling technique reveals that autonomic neurons innervated by the same axon project to the same target region}

Shlomo Tsuriel, Sagi Gudes, Ryan W Draft, Alexander M. Binshtok, Jeff W. Lichtman

\section{Supplementary Information}

Supplementary Movie 1. Three dimensional rendering of a confocal stack showing the location of all the vesicles in a ganglion cell. Shown are red and green vesicles following two injections to near but non-overlapping parts of the gland. Note that red and green vesicles are common but by rotation it can be seen that there are no yellow vesicles that share both colors.

Supplementary Figure 1. Preganglionic input, rather than ganglion cell body location, correlates with axonal arbor position. (a) The average distance between the axonal projections in the submandibular gland of pairs of ganglion cells that are innervated by the same preganglionic axon (red column) compared to pairs of neurons that are innervated by different inputs (white column). The difference is significant; $p<0.001$. (b) However, within the ganglion cell cluster, the average distance between the pairs of somata themselves is roughly the same for the neurons that share the same input (red column) versus those that don't (white column). (c) Moreover when we looked at each of 515 pairs of neurons individually we found little correlation between the distances between somata and distances between axon projections pairs $(r=0.0092)$. Although pairs of ganglion cells that share common input (red circles) did tend to be closer to each other in the gland. (d) The separation of arbors within the gland is also affected by the degree to which two ganglion cells share the same axonal input. In cases where one ganglion cell of the pair is innervated by more than one preganglionic axon (gray column) the arbors are significantly more distant than ganglion cells pairs that have identical input (red column) but significantly closer than pairs that do not share any preganglionic input (white column). Data combined from P32 and P18 ganglia. Student $\mathrm{t}$ test; $*=\mathrm{p}<0.05 ; * *=\mathrm{p}<0.01 ; * * *=\mathrm{p}<0.001 ; \#=$ $\mathrm{p}>0.05$. 
